\title{
COMMENT \\ CD26 - The emerging role of a costimulatory molecule in allograft rejection
}

\author{
Cellular \& Molecular Immunology (2020) 17:1208-1209; \\ https://doi.org/10.1038/s41423-018-0028-9
}

Rejection after transplantation (Tx) between MHC disparate individuals remains an unresolved issue. Among many cells of the innate and adaptive immune system, $T$ cells are mainly responsible for the allorejection process. Three signals are required for full T-cell activation: (I) antigen-specific T-cell receptor engagement, (II) a costimulatory signal, and (III) the production of signature cytokines produced by both engaged antigen-presenting cells and T cells, thus inducing optimal T-cell proliferation. Isolated TCR-MHC interactions actually lead to T-cell unresponsiveness rather than activation, otherwise termed anergy, and most importantly, contact between a costimulatory molecule on the T-cell and its ligand on functional DCs is necessary for the full activation and proliferation of T cells. CD28 is probably the most commonly cited example of these costimulatory receptors, but other related molecules that provide costimulatory signals during T-cell activation have since been identified and are collectively referred to as 'positive' costimulatory receptors and ligands.

One of these receptors that possess costimulatory properties is CD26, a molecule that is abundantly expressed on hematopoetic cells as well as epithelial cells, endothelial cells, and fibroblasts. The complete amino acid sequence of human CD26 was first published in $1992 .^{1}$ In addition to its costimulatory property, this molecule has enzymatic activity (DPP IV) and cleaves peptides at their penultimate position (Fig. 1A). Upon CD26-mediated costimulation, IL-2 production is increased in cells expressing wild-type CD26, suggesting that the DPP IV enzymatic activity of CD26 may contribute to signaling. ${ }^{2}$ Inhibiting the enzymatic activity of CD26 through specific inhibitors suppresses T-cell proliferation in vitro and decreases antibody production in mice immunized with bovine serum albumin. ${ }^{3}$ The role of CD26 after alloantigen exposure in vivo triggered by organ transplantation was studied by inhibiting CD26 in the immune cascade. This inhibition resulted in an abrogation of acute rejection and prolongation of allograft survival in a rat cardiac transplantation model. $^{4}$ Furthermore, suppression of systemic CD26/DPP IV enzyme activity was associated with severely impaired host cytotoxic T lymphocyte responses in vitro. ${ }^{4}$ Moreover, CD26inhibited animals lacked the allospecific lgM response and had greatly decreased IgG serum titers. ${ }^{5}$ Therefore, inhibition of the enzymatic activity of CD26/DPP IV is thought to interfere with the early steps in the humoral response to an alloantigen after organ transplantation. Recent studies have also focused on the kidney as the main organ of study for CD26 in the context of post-transplant rejection ${ }^{6}$ or as a biomarker for assessing adequate immunosuppression after kidney Tx. ${ }^{7}$ Inhibiting the action of CD26 has also been analyzed in graft vs. host disease. ${ }^{8}$ Recently, our own group has shown the potential of inhibiting the costimulatory action of CD26 by the CD26 inhibitor Vildagliptin. Inhibition of CD26 resulted in attenuation of rejection by suppressing T-cell activation in experimental mouse lung Tx. ${ }^{9}$
In the present study by Zhao, Fan and colleagues, authors investigate the role of CD26 as a costimulatory molecule for T-cell activation after skin allotransplantation. ${ }^{10}$ In a diverse and well-balanced set of experiments using the MHC class I and class II mismatched mouse strain combination of BALB/C as donors and C57BL/6 as transplant recipients, authors demonstrate that transplantation of skin allografts into CD26 knockout (k. o.) mice result in delayed graft rejection showing less skin graft loss in these mice, in parallel with diminished infiltration of $\mathrm{CD}^{+}$and $\mathrm{CD}^{+} \mathrm{T}$ cells and macrophages as well as fewer $\mathrm{CD}^{+} \mathrm{T}$ cells in peripheral blood. By further dissecting the T-cell compartment, authors found fewer Th17 cells, but an increased number of regulatory $T$ cells within transplants. These data strongly favor an immunosuppressive environment within these CD26 k. o. mice and were further supported by the observation that a series of pro-inflammatory cytokines were found to be decreased while IL-10 cytokine levels were increased in CD26 k. o. transplant recipients. Most remarkably, authors found reduced lgG1 levels in the serum of CD26 k. o. mice, although the total B cell count remained unchanged within recipients. This finding suggests that the release of $\lg 1$ is dependent on CD26 signaling, supporting the notion that $C D 26^{\text {bright }} T$ cells are not only important for recruiting $T$ cells for $B$ cell $\mathrm{lg}$ synthesis, but also that CD26 is expressed on B cells in wild-type mice. Together, these data impressively support the hypothesis of the immunomodulatory function of CD26 in allotransplantation towards an immunosuppressive milieu (Fig. 1B). Given that the authors chose a skin transplant model, the results are even more remarkable as the skin is one of the most immunogeneic organs harboring large numbers of antigen-presenting (dendritic) cells.

In most studies addressing the role of CD26, an inhibitor of CD26 is used. In the present study, authors chose a CD26 k. o. strain a priori to demonstrate its effects. It would be interesting to see if similar effects would occur when using a CD26 inhibitor, e.g., vildagliptin or the even more DPP IV - specific inhibitor Sitagliptin. The CD26 k. o. model provides an ideal experimental setup; however, inhibition of CD26 is only possible in the clinical setting by pharmaceutical modulation. The data provided by the authors are compelling and suggest that the development, maturation, and function of CD26-bearing cells are profoundly impaired. Although the absence of CD26 can abolish rejection via downregulation of inflammatory cells of the innate and adaptive immune system, the mechanisms of how these effects are exerted remain largely unclear from this study. For example, is the reduced level of IgG1 the result of impaired activation and differentiation of $B$ cells due to lower levels of cytokines such as IFN- $\gamma$ or IL-2, or is it rather the effect of diminished expression of the CD26 receptor on B cells? Furthermore, it is unclear whether and which of the observed effects result from inhibition of costimulation vs. inhibition of the enzymatic activity of CD26/DPP IV and to what extent the enzymatic activity impacts the costimulatory activity, and vice versa. Finally, it is questionable whether these results can be translated to other solid transplantable organs as the skin is not a solid organ. Yet, the authors need to be commended on this 
A enzymatic activity (DPPIV)

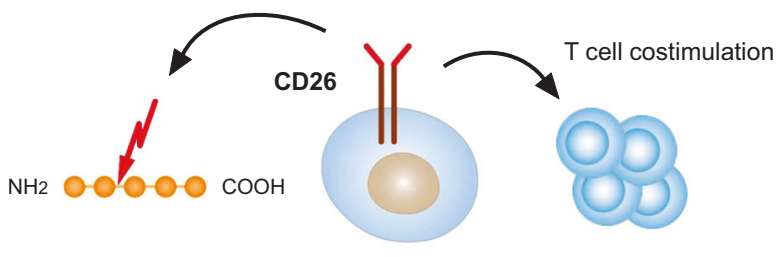

B

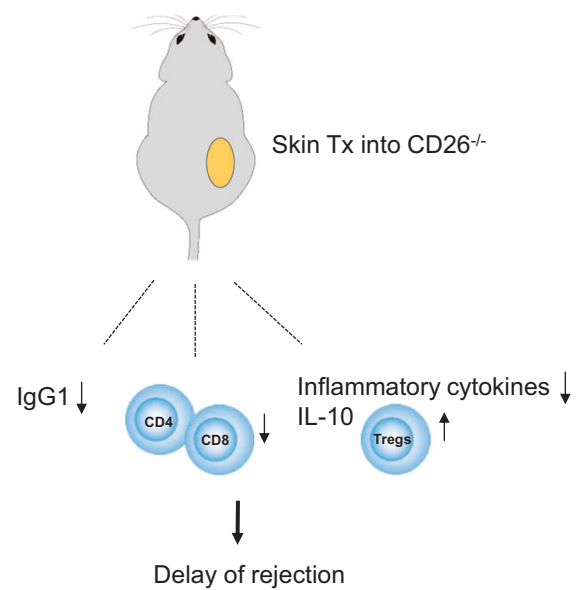

Fig. 1 CD26/DPP IV has enzymatic and costimulatory activities. The enzymatic activity of DPP IV cleaves peptides at their penultimate position, and the costimulatory activity of CD26 signals activation and proliferation of $T$ cells $(A)$. Upon skin transplantation from $\mathrm{BALB} / \mathrm{c}$ into $\mathrm{CD} 26 \mathrm{k}$. o. mice on a $\mathrm{C} 57 \mathrm{BL} / 6$ background, reduced levels of IgG1 antibodies were found. The number and function of $\mathrm{CD}^{+}$and $\mathrm{CD}^{+}$cells were decreased and reduced levels of pro-inflammatory cytokines such as IL-2, IFN- $\gamma$, and IL- 6 were found. By contrast, IL-10 levels were enhanced and the number of regulatory $T$ cells was increased. All of these findings lead to a delay in allograft rejection (B)

piece of work, as it provides further evidence on the importance of this costimulatory molecule and its implications in transplantation medicine. Although human CD26 shares $~ 85 \%$ homology with mouse CD26, the differences between human and rodent CD26 regarding its ability for costimulation limits the utility of murine models for elucidating the function of human CD26. On the other hand, CD26-specific inhibitors have been in use over a decade as anti-diabetic drugs and could be repurposed for novel immunomodulatory transplant protocols in clinical practice.

\section{ADDITIONAL INFORMATION}

Competing interests: The authors declare no competing interests.

W Jungraithmayr ${ }^{1}$ and N Enz ${ }^{1}$
${ }^{1}$ Department of Thoracic Surgery, Brandenburg Medical School,
Neuruppin, Germany

\section{REFERENCES}

1. Darmoul, D. et al. Dipeptidyl peptidase IV (CD 26) gene expression in enterocytelike colon cancer cell lines HT-29 and Caco-2. Cloning of the complete human coding sequence and changes of dipeptidyl peptidase IV mRNA levels during cell differentiation. J. Biol. Chem. 267, 4824-4833 (1992).

2. Morimoto, C. \& Schlossman, S. F. The structure and function of CD26 in the T-cell immune response. Immunol. Rev. 161, 55-70 (1998).

3. Kubota, T. et al. Involvement of dipeptidyl peptidase IV in an in vivo immune response. Clin. Exp. Immunol. 89, 192-197 (1992).

4. Korom, S. et al. Inhibition of CD26/dipeptidyl peptidase IV activity in vivo prolongs cardiac allograft survival in rat recipients. Transplantation 63, 1495-1500 (1997).

5. Korom, S. et al. Specific inhibition of CD26/DPP IV enzymatic activity in allograft recipients: effects on humoral immunity. Transplant. Proc. 31, 778 (1999).

6. Leicht, S. et al. CD26/dipeptidyl peptidase IV: a comparative study of healthy persons and kidney transplant recipients before and early after transplantation. Clin. Biochem. 46, 1383-1388 (2013).

7. Wieland, E. et al. Association between pharmacodynamic biomarkers and clinical events in the early phase after kidney transplantation: a single-center pilot study. Ther. Drug. Monit. 33, 341-349 (2011).

8. Hatano, R. et al. Prevention of acute graft-versus-host disease by humanized antiCD26 monoclonal antibody. Br. J. Haematol. 162, 263-277 (2013).

9. Yamada, Y. et al. CD26 costimulatory blockade improves lung allograft rejection and is associated with enhanced interleukin-10 expression. J. Heart Lung. Transplant. 35, 508-517 (2016).

10. Zhao, X. Z. K., Daniel, P., Wisbrun, N., Fuchs, H., Fan, H. Delayed allogeneic skin graft rejection in CD26-deficient mice. Cell. Mol. Immunol. (2018) in press. 